\title{
Differential response to gefitinib of cells expressing normal EGFR and the mutant EGFRvill
}

\author{
MW Pedersen', N Pedersen', LH Ottesen ${ }^{2}$ and HS Poulsen*,I \\ 'Department of Radiation Biology, The Finsen Center, Copenhagen University Hospital, Blegdamsvej 9, Copenhagen DK-2 I00, Denmark; ${ }^{2}$ Medical \\ Department, AstraZeneca, Roskildevej 22, Albertslund DK-2620, Denmark
}

\begin{abstract}
Epidermal growth factor receptor (EGFR) is frequently amplified and/or mutated in a number of human tumours and abnormal signalling from this receptor is believed to contribute to the malignant phenotype seen in these tumours. Gefitinib is a small molecule inhibitor that specifically binds and inhibits the EGFR tyrosine kinase and has been shown to inhibit the growth, proliferation, survival and invasion of a range of tumour cells overexpressing EGFR. However, clinical response to gefitinib has failed to correlate with EGFR levels and activity, indicating that other molecular mechanisms such as downstream signalling and mutations could be of importance in predicting clinical response. We therefore investigated the effect of the specific EGFR inhibitor gefitinib on the phosphorylation level, signalling and growth of cells expressing the naturally occurring constitutively active EGFR variant EGFRvIll, a low nontransforming level of EGFR and a high transforming level of EGFR. Results show that levels of gefitinib sufficient to suppress EGFR phosphorylations, EGFR-mediated proliferation and EGFR-mediated anchorage-independent growth are not sufficient to inhibit these features in cells expressing EGFRvIII. Furthermore, the data indicate that long-term exposure of EGFRvIll-expressing cells to low concentrations of gefitinib $(0.01-0.1 \mu \mathrm{M})$ result in increased phosphotyrosine load of the receptor, increased signalling to ERK and stimulation of proliferation and anchorage-independent growth, presumably by inducing EGFRvlll dimerisation. Higher concentrations of gefitinib $(\mathrm{I}-2 \mu \mathrm{M})$, on the other hand, significantly decreased EGFRvIII phosphotyrosine load, EGFRvIII-mediated proliferation and anchorageindependent growth. Further studies are needed to investigate the implications of these important findings in the clinical setting. British Journal of Cancer (2005) 93, 915-923. doi: I 0.1038/sj.bjc.6602793 www.bjcancer.com
\end{abstract}

Published online 27 September 2005

(c) 2005 Cancer Research UK

Keywords: EGFR; EGFRvIll; iressa; gefitinib; signalling; mutation

Receptor tyrosine kinases regulate signalling pathways involved in critical cellular activities such as growth, proliferation, motility, survival and apoptosis. When activated by overexpression, autocrine growth factor stimulation or mutations, receptor tyrosine kinases can contribute to the development of human cancers. The epidermal growth factor receptor (EGFR, ErbB1) is a tyrosine kinase receptor of the ErbB family and is frequently overexpressed or mutated in human malignancies including those of the brain, breast, colon, ovary and lung (Tang et al, 2000; Alper et al, 2001; Ge et al, 2002; Peghini et al, 2002). This has inspired the development of specific pharmacological inhibitors of the EGFR tyrosine kinase such as gefitinib (Iressa, AstraZeneca Pharmaceuticals), which disrupts EGFR kinase activity by reversibly binding within the ATP-binding pocket of the EGFR protein (Arteaga and Johnson, 2001). Gefitinib is orally active and has shown promising antitumour activity in vitro and in vivo, although clinical response has failed to correlate with either EGFR levels or activity. Recently, however mutations were identified in the tyrosine kinase domain, which were associated with sensitivity of non-small-cell lung cancer to gefitinib (Lynch et al, 2004; Paez et al, 2004).

*Correspondence: Dr HS Poulsen; E-mail: skovgaard@rh.dk Received 28 February 2005; revised 7 July 2005; accepted 23 August 2005; published online 27 September 2005
The type III epidermal growth factor mutation variously named, EGFRvIII, $\triangle$ EGFR, de2-7EGFR and mEGFR is the most common mutation in the EGFR gene and is frequently found in tumours of the breast, ovary, prostate, lung and in particular those of the brain (Garcia et al, 1993; Moscatello et al, 1995; Olapade-Olaopa et al, 2000; Pedersen et al, 2001; Ge et al, 2002). This mutation or deletion eliminates exons 2-7 resulting in a truncated receptor with a distorted ligand-binding area. However, despite lack of ligand-binding EGFRvIII has a constitutively active receptor tyrosine kinase and is able to transform fibroblasts and to confer enhanced tumorigenicity to cancer cells both in vitro and in vivo (Huang et al, 1997; Damstrup et al, 2002; Pedersen et al, 2004). A recent study found that cells expressing EGFRvIII had increased resistance to gefitinib in vitro and in vivo, which were attributed to a deficiency in receptor dephosphorylation and constitutive AKT activity (Learn et al, 2004). Hence, evidence accumulates that signalling regulated by EGFR is altered by overexpression or mutations and that such alterations can lead to differences in response to gefitinib treatment.

The purpose of this study was to investigate the effect of gefitinib on phosphorylation level, intracellular signalling and growth properties of cells expressing EGFRvIII. However, there are several complicating factors associated with the analysis of EGFRvIII-mediated signalling in cancer cells. In particular, the enhanced tumorigenicity conferred to cancer cells by EGFRvIII is 
predominantly restricted to in vivo growth conditions making detailed analyses problematic. Many cancer cells also express significant levels of other members of the ErbB family that are potentially capable of forming heterodimers with EGFRvIII (O’Rourke et al, 1998).

Therefore we have used the unique NR6 model system, in which NR6wtEGFR express a low nontransforming level of EGFR and the fully tumorigenic cell lines NR6W and NR6M that overexpress EGFR or express mutant EGFRvIII, respectively (Batra et al, 1995). This allows us to characterise the effect of gefitinib on phosphorylation and intracellular signalling by a nontransforming level of EGFR, by a transforming level of EGFR and by a transforming level of EGFRvIII.

Results show that some tyrosine phosphorylation sites on the wild-type (wt)-EGFR are more resistant to inhibition by gefitinib compared to others. Results also confirm that higher doses of gefitinib are needed to abrogate phosphorylation of and signalling to PLC- $\gamma$ and AKT by EGFRvIII as compared to wt-EGFR. Furthermore, small levels of gefitinib seem to induce receptor autophosphorylation, as well as proliferation and anchorageindependent growth of EGFRvIII-expressing cells.

\section{MATERIALS AND METHODS}

\section{Materials}

Recombinant human EGF was purchased from Calbiochem (Germany). Anti EGFR, phospho-EGFR (Tyr845, Tyr992, Tyr1045, Tyr1068), STAT3, phospho-STAT3 (Tyr705), AKT, phospho-AKT (Ser473), extracellular-regulated kinase (ERK)-1/2 and phospho-ERK-1/2 (Thr202/Tyr204) antibodies were from Cell Signaling Technology (Germany). Antiphospho-EGFR (Tyr1148 and Tyr1173) antibodies were from Upstate Biotechnology (USA). The antiphospho-EGFR (Tyr1086) antibody was from Biosource (USA).

Antibody to tubulin was from Santa Cruz Biotechnology Inc. (CA, USA). HRP-conjugated secondary antibodies were purchased from, DAKO (Denmark).

\section{Cell lines}

Four cell lines were included in this work. The cell lines NR6, NR6M and NR6W have been described previously and were kindly provided by Dr Darell Bigner, Duke University, NC, USA (Batra et al, 1995). The NR6wtEGFR cell line, which expresses a lower number of receptors as compared to NR6W, has also been described previously and is a generous gift from Dr Allan Wells, Department of Pathology, University of Pittsburgh (Wells et al, 1990).

\section{Immunoblot analyses}

For determination of phosphorylated proteins, $5 \mu \mathrm{g}$ whole cell lysate from serum-starved cells was resolved by SDS-PAGE and electroblotted onto nitrocellulose membranes. After transfer and blocking in 5\% nonfat milk, primary antibody staining was carried out by incubation overnight at $4{ }^{\circ} \mathrm{C}$, and secondary antibody staining was for $1 \mathrm{~h}$ at room temperature. The chemiluminescence detection method (ECL) was used for all Western blot experiments.

\section{Crosslinking assay}

Crosslinking of receptors were carried out as described (Montgomery, 2002). Briefly, gefitinib-treated cells were washed twice in ice-cold phosphate-buffered saline (PBS) and solubilised in RIPA buffer containing protease and phosphatase inhibitors, $10 \%$ glycerol and $1 \mathrm{mM}$ bis(sulfosuccinimidyl) suberate $\left(\mathrm{BS}^{3}\right)$ for $20 \mathrm{~min}$ at $4^{\circ} \mathrm{C}$. Glycine at a final concentration of $250 \mathrm{~mm}$ was subsequently added for $5 \mathrm{~min}$, followed by centrifugation at $14000 \mathrm{~g}$ for $10 \mathrm{~min}$. Equivalent amounts of protein were resolved by SDS-PAGE and electroblotted onto nitrocellulose membranes. Blotting and antibody incubations were performed as above using anti-EGFR and antiphospho-tyrosine antibodies.

\section{Proliferation assay}

Exponentially growing cells were seeded in sextuple in 96-well plates at a concentration of 2000 cells/well, allowed to adhere and subsequently washed in PBS and incubated overnight in medium containing $0.5 \%$ FCS. Cells were then treated with varying concentrations of Iressa or the solute control DMSO and EGF. The optimal EGF concentration for inducing proliferation of NR6wtEGFR and NR6W cells has previously been determined and hence NR6wtEGFR and NR6W cells were added 10 and $0.1 \mathrm{~nm}$ EGF, respectively (Pedersen et al, unpublished observation). NR6 and NR6M cells were not added EGF. After $72 \mathrm{~h}$ the amount of cells were measured by performing a 3-[4,5-dimethylthiazol-2-yl]-2,5diphenyltetrazolium bromide (MTT) proliferation assay (Roche, Denmark) (Hansen et al, 2003).

\section{Soft agar assay for anchorage-independent growth}

Exponentially growing cells $\left(1 \times 10^{5}\right)$ were suspended in $3 \mathrm{ml} 0.5 \%$ (w/v) NuSieve low-melting agar (FMC, Rockland, ME, USA) dissolved in DMEM $+0.5 \%$ FCS and plated in six-well plates covered with $0.5 \%$ agar dissolved in DMEM $+0.5 \%$ FCS. Cells were then treated with varying concentrations of Iressa or the solute control DMSO. The optimal concentration of EGF for inducing anchorage-independent growth of NR6wtEGFR and NR6W cells has previously been determined and hence NR6wtEGFR were added 10 and 0.1 nM EGF, respectively (Pedersen $e$ t al, unpublished observation). NR6 and NR6M were not stimulated with EGF. Cultures in triplicate for each condition were replenished with fresh medium once a week. After 3 weeks the plates were stained with crystal violet and colonies $>50$ cells were counted.

\section{RESULTS}

\section{Expression of ErbB family members in the cell lines}

Epidermal growth factor receptor and EGFRvIII are known to form heterodimers with other members of the ErbB receptor family, which could potentially affect receptor phosphorylation and downstream signalling. Thus, the levels of ErbB1 (EGFR), ErbB2 (HER-2), ErbB3 (HER-3) and ErbB4 (HER-4) in the four NR6 cell lines: NR6, NR6wtEGFR, NR6W and NR6M were investigated by immunoblotting (Figure 1). All four cell lines have comparable levels of ErbB2, ErbB3 and ErbB4, which appear to be relatively low as compared to the levels of EGFR and EGFRvIII. Consequently, heterodimerisation most likely play a minor role in these cell lines.

\section{Effect of gefitinib on inhibition of EGFR tyrosine phosphorylations}

To investigate the efficacy of gefitinib towards inhibition of wt and mutant EGF receptors, we evaluated the effect of varying concentrations $(0.001-2 \mu \mathrm{M})$ of the inhibitor on receptor phosphorylations in the cell lines: NR6wtEGFR, NR6W and NR6M by immunoblotting (Figure 2). Initially cells were serum starved overnight to reduce baseline levels of phosphorylation. The cells were then mock treated (DMSO only) or treated with increasing concentrations of gefitinib for $5 \mathrm{~h}$, after which they were stimulated with $10 \mathrm{nM}$ of EGF for $10 \mathrm{~min}$.

Immunoblot analysis of whole cell lysates revealed that in general gefitinib effectively inhibited all tyrosine phosphorylation sites on EGFR in both the high and low-EGFR-expressing cell lines 
(Figure 2A and B). However, the phosphorylation sites Tyr1173 and Tyr992 were less sensitive requiring higher concentrations of gefitinib for inhibition. The calculated $\mathrm{IC}_{50}$ values for these sites were $37 \mathrm{~nm}$ (Tyr1173), $37 \mathrm{~nm}$ (Tyr992), $26 \mathrm{~nm}$ (Tyr1173) and $57 \mathrm{~nm}$

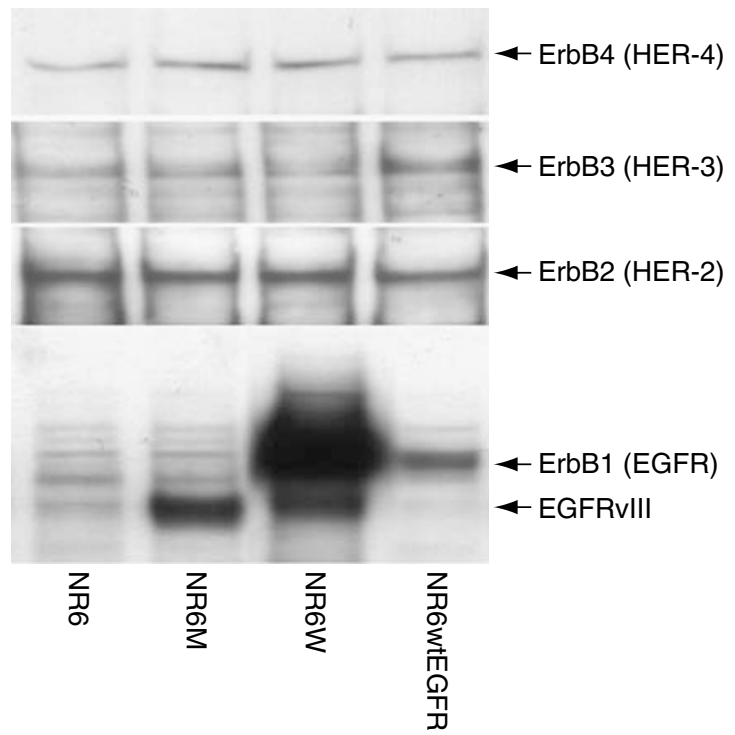

Figure I Expression of the ErbB family members in the cell lines described in these experiments as measured by immunoblotting using specific antibodies.
(Tyr992) in, respectively, the low and high EGFR expressing cell lines (Table 1).

The tyrosine phosphorylation sites in the EGFRvIII-expressing cell line, although sensitive to gefitinib, were all very durable requiring 3-20 times higher doses for 50\% inhibition as compared to the wt-EGFR (Figure 2C and Table 1). The Tyr1173 and Tyr1148 were the sturdiest sites with $\mathrm{IC}_{50}$ values of 97 and $214 \mathrm{nM}$, respectively. Thus, although EGFRvIII has a lower level of phosphorylation as compared to ligand-activated EGFR; the phosphotyrosine residues are more resistant to inhibition by gefitinib. Although an isolated increase in phosphorylation of EGFRvIII on some tyrosine residues was noted in the NR6M cells treated with 0.005 and $0.01 \mu \mathrm{M}$ (Figure 1C, lanes 4 and 5), this result was not consistently seen on repeat experiments.

The average $\mathrm{IC}_{50}$ values for tyrosine phosphorylation of EGFR in the low- and high-expressing cell lines were 22 and $21 \mathrm{~nm}$, respectively, and thus independent of receptor expression levels. In contrast the average IC $_{50}$ value of EGFRvIII tyrosine phosphorylation was $84 \mathrm{~nm}$, suggesting that the EGFRvIII tyrosine kinase is approximately four times as resistant to gefitinib as EGFR (Table 1).

\section{Differential inhibition of intracellular signalling by gefitinib}

The effect of gefitinib on the status of the major downstream targets of EGFR and EGFRvIII were also investigated. Extracellular regulated kinase-1 and -2 (ERK-2), phospholipase C $\gamma$ (PLC- $\gamma$ ), protein kinase $\mathrm{B}(\mathrm{AKT})$ and the signal transducer and activator of transcription 3 (STAT3) are all essential downstream targets of EGFR and mediate many of its oncogenic effects.
A
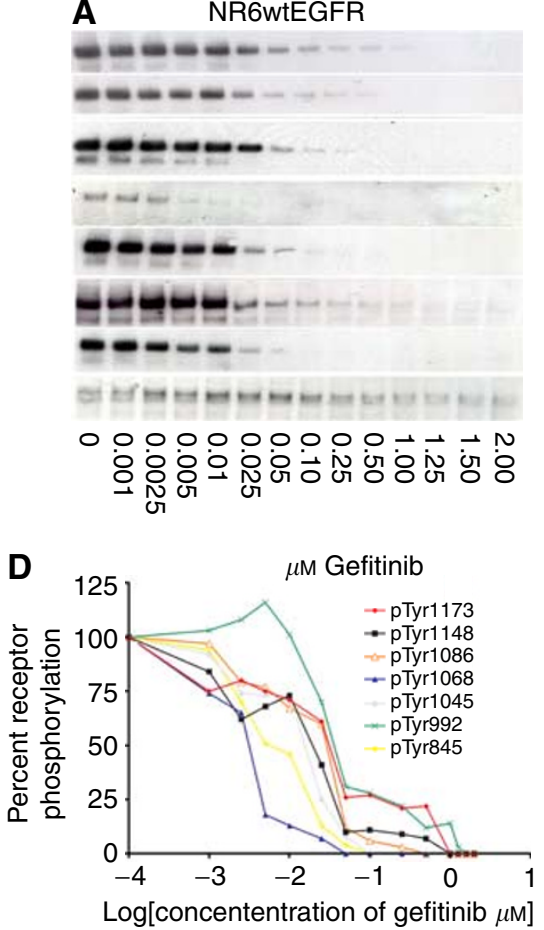

B

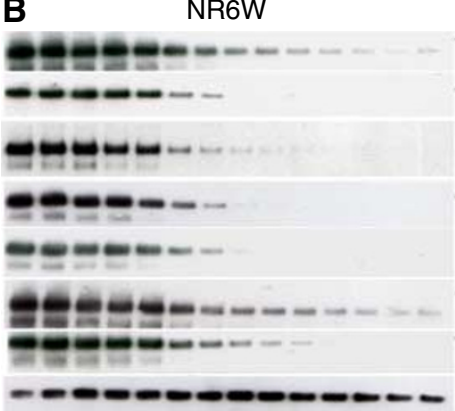

$0000000000 \rightarrow-\rightarrow N$

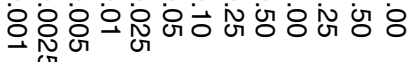

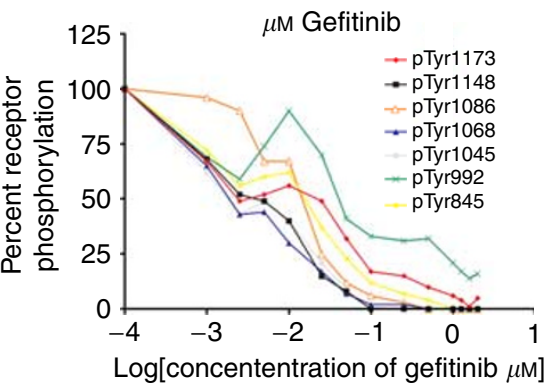

C
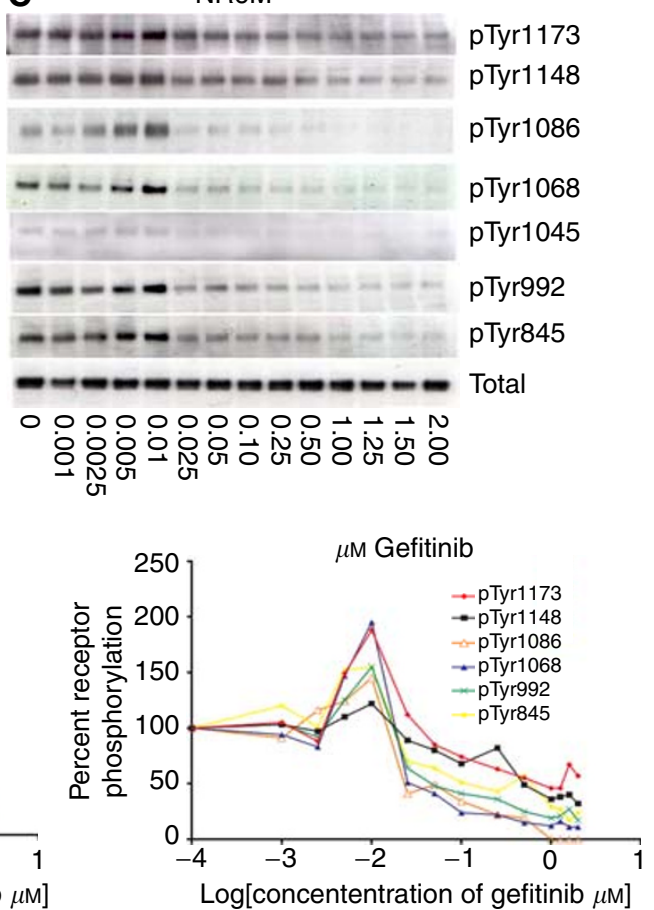

Figure 2 Profiles of EGF- (IO nM for $10 \mathrm{~min}$ ) induced phosphorylation of various tyrosine residues of EGFR in the presence of varying concentrations of gefitinib in (A) the low-EGFR-expressing cell line NR6wtEGFR and (B) the high-EGFR-expressing cell line NR6W. (C) Profiles of constitutive phosphorylation of various tyrosine residues of EGFRvIll in the presence of varying concentrations of gefitinib. The phosphorylations were determined by immunoblotting of whole cell lysates with antibodies that specifically recognise the phosphorylated amino-acid residues Tyr845, Tyr992, Tyr 1045 , Tyr 1068 , Tyr 1086, Tyrl I 48 and Tyrl | 73. Total EGFR or EGFRvlll levels are also indicated. (D) Bands were quantified using Kodak Digital Science Software version 1.0 corrected for total receptor level and plotted as a percentage of the phosphorylation levels in the untreated cells (lower panel). Exposures of the various blots are optimised for quantitative detection of $\mathrm{IC}_{50}$ and thus not directly comparable. 
Table I $\quad C_{50}(\mathrm{nM})$ of specific EGFR tyrosine phosphorylation site inhibition by gefitinib

\begin{tabular}{lcccccccc}
\hline & \multicolumn{7}{c}{ EGFR tyrosine phosphorylation sites } \\
\cline { 2 - 8 } Cells & Tyrl I 73 & Tyrl I 48 & Tyr 1086 & Tyr 1068 & Tyr 1045 & Tyr992 & Tyr845 & Average \\
\hline NR6wtEGFR & 37 & 17 & 20 & 2 & 13 & 37 & 6 \\
NR6W & 26 & 5 & 12 & 3 & 4 & 57 & 18 & 22 \\
NR6M & 97 & 214 & 47 & 45 & - & 47 & 52 \\
\hline
\end{tabular}

\section{A

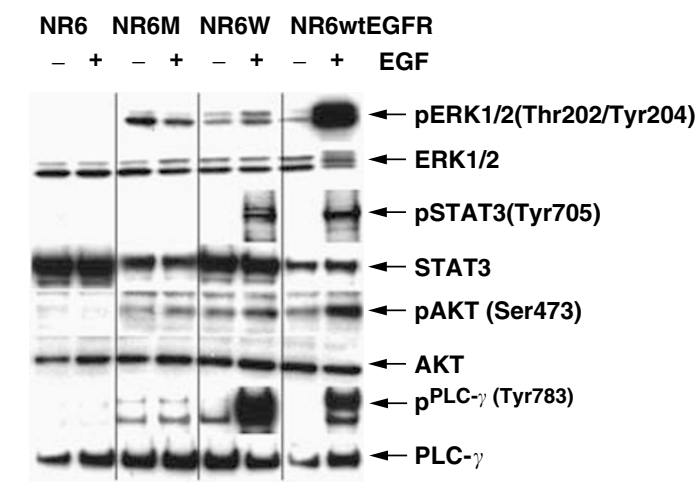

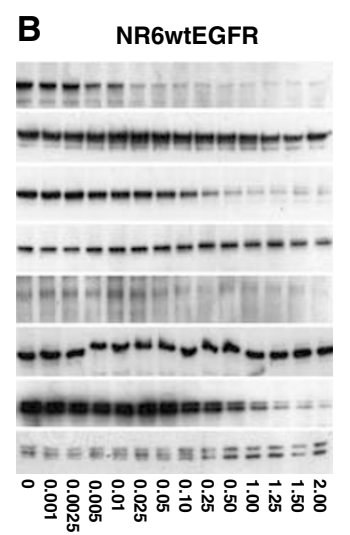

$\mu \mathrm{M}$ Gefitinib

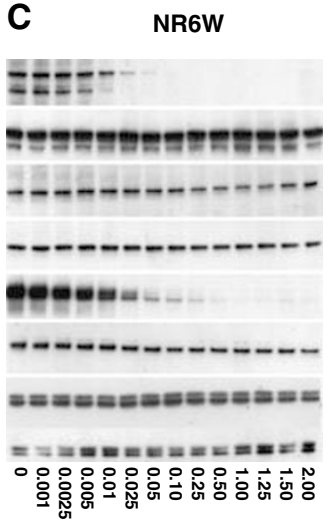

$\mu \mathrm{M}$ Gefitinib

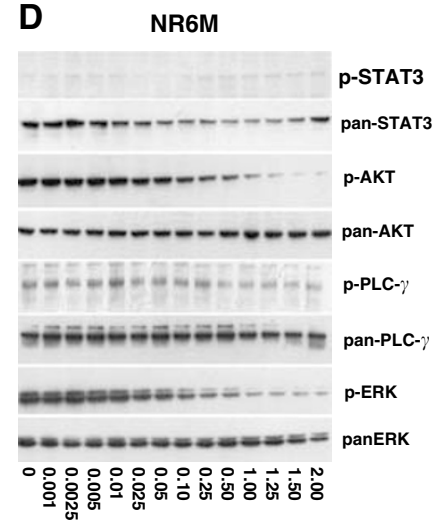

$\mu \mathrm{M}$ Gefitinib

Figure 3 (A) Phosphorylation of key signalling molecules upon EGF ( $10 \mathrm{~nm}$ for $10 \mathrm{~min}$ ) stimulation in the four cell lines. Profiles of EGF- ( $10 \mathrm{~nm}$ for $10 \mathrm{~min}$ ) induced phosphorylation of various downstream signalling molecules of EGFR in cells pretreated with varying concentrations of gefitinib in (B) the lowEGFR-expressing cell line NR6wtEGFR and $(\mathbf{C})$ in the high-EGFR-expressing cell line NR6W. (D) Profiles of EGFRvlll-induced phosphorylation of various downstream signalling molecules of EGFR in the presence of varying concentrations of gefitinib in the EGFRvIll-expressing cell line NR6M. The phosphorylations and total levels of the signalling molecules were determined by immunoblotting of whole cell lysates with antibodies that specifically recognise the total and phosphorylated species of ERK, PLC- $\gamma$, AKT and STAT3.

EGF-induced phosphorylation of ERK in the low EGFRexpressing cell line, but not significantly in the high-EGFRexpressing cell line (Figure 3A). The EGFRvIII-expressing cell line had a low level of constitutively phosphorylated ERK as a result of the constitutively active receptor (Figure $3 \mathrm{~A}$ ). Using a concentration gradient it was found that higher concentrations of gefitinib were needed to inhibit ERK signalling ( $\mathrm{IC}_{50}$ of 394 and $356 \mathrm{nM}$, respectively) as compared to the receptor phosphorylations ( $\mathrm{IC}_{50}$ of 22 and $84 \mathrm{nM}$, respectively) in the NR6wtEGFR and NR6M cell lines (Table 2, Figure 3B and D). ERK phosphorylations in the NR6W cell line in contrast were unresponsive to gefitinib even at the highest concentrations indicating that the ERK activity in this cell line is not mediated by the EGFR (Figure 3C).

As opposed to the level of ERK phosphorylation the high EGFR-expressing cell line had a substantial level of PLC- $\gamma$ phosphorylation after EGF stimulation as compared to the low-EGFRand -EGFRvIII-expressing cell lines, respectively (Figure 3A). Gefitinib
Table $2 \quad I_{50}(n M)$ of specific signalling molecule phosphorylation site inhibition by gefitinib

\begin{tabular}{lccccc}
\hline & \multicolumn{5}{c}{ Inhibition of phosphorylation sites } \\
\cline { 2 - 6 } Cells & PLC- $\boldsymbol{\gamma}$ & AKT & ERK & STAT3 & Average \\
\hline NR6wtEGFR & 43 & 220 & 394 & 17 & 169 \\
NR6W & 27 & 7 & - & 8 & 11 \\
NR6M & 369 & 263 & 356 & - & 329 \\
\hline
\end{tabular}

effectively blocked this phosphorylation with a calculated $\mathrm{IC}_{50}$ of $27 \mathrm{~nm}$ (Table 2 and Figure 3C). The NR6wtEGFR and NR6M cell lines had low levels of PLC- $\gamma$ phosphorylations but the level in the $\mathrm{NR} 6 \mathrm{M}$ cell line was more resistant to inhibition by gefitinib $\left(\mathrm{IC}_{50}\right.$ of 43 and $369 \mathrm{~nm}$, respectively) see Table 2, Figure 3B and D. 
The levels of AKT phosphorylation are most considerable in the low-EGFR- and -EGFRvIII-expressing cell lines and unresponsive to EGF in the high-EGFR- and -EGFRvIII-expressing cell lines (Figure 3A). As was the case for ERK, gefitinib fails to effectively inhibit AKT phosphorylation in the high-EGFR-expressing cell line indicating that EGFR is not the major activator of AKT in this cell line. The low $\mathrm{IC}_{50}(7 \mathrm{nM})$, however show that the weak induction of AKT phosphorylation by EGFR in this cell line is efficiently blocked by gefitinib (Table 2). Gefitinib inhibits AKT phosphorylations, with $\mathrm{IC}_{50}$ values of 220 and $263 \mathrm{nM}$, in the low-EGFR- and -EGFRvIII-expressing cell lines, respectively (Table 2, Figure 3B, C and D). Only the wt receptor is able to activate STAT3 upon EGF stimulation of both the high- and low-EGFR-expressing cell lines (Figure 3A). Of the downstream targets of EGFR, STAT3 seems to be most dependent on high receptor phosphorylation. STAT3 phosphorylation is completely abolished at $\mathrm{IC}_{50}$ values of 17 and $8 \mathrm{nM}$, which is comparable to the levels needed for inhibition of receptor autophosphorylation (Table 2, Figure $3 \mathrm{~B}$ and $\mathrm{C}$ ). There is no effect of gefitinib on the very low background level of STAT3 phosphorylation in the EGFRvIII-expressing cell line.

\section{Inhibition of EGFRvIII driven cell proliferation requires higher concentrations of gefitinib than wt EGFR}

The rate of proliferation in the absence of gefitinib was higher in the EGFR- and EGFRvIII-expressing cell lines, as compared to the
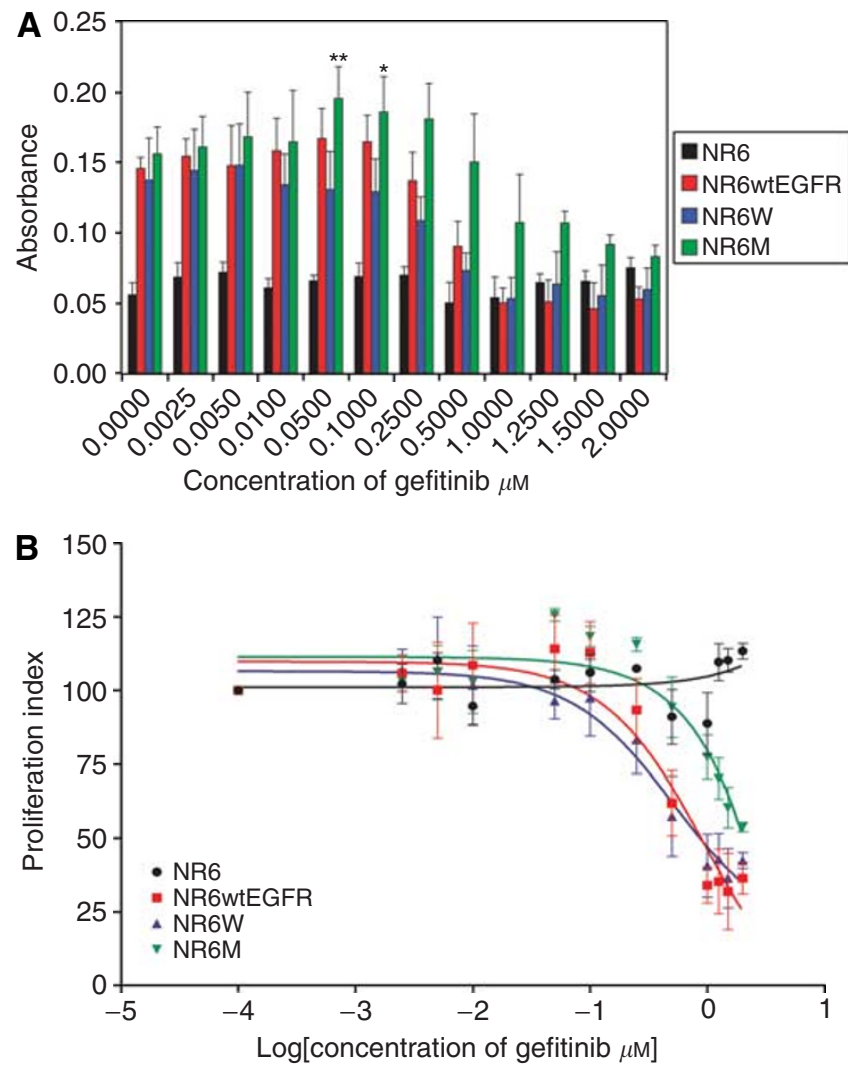

Figure 4 Effect of gefitinib on cell proliferation. (A) Graphical presentation of raw absorbance measurements for NR6, NR6wtEGFR, NR6W and NR6M cells treated with $0-2 \mu \mathrm{M}$ gefitinib for $72 \mathrm{~h}$. NR6, NR6wtEGFR and NR6W cells were stimulated with EGF. This figure is representative of 15 independent experiments; bars, \pm s.e. $* P<0.05$ (compared to control treatment), ${ }^{*} * P<0.01$ (compared to control treatment). (B) Proliferation plotted as a percentage of the viability in the untreated cells; bars, \pm s.e. parental cell line NR6 (Figure 4A). Gefitinib had no effect on NR6 proliferation in the concentration range investigated. In contrast, proliferation of cells expressing EGFR decreased significantly, reaching a level similar to the parental cell line at a gefitinib concentration of $1 \mu \mathrm{M}$. Proliferation of cell expressing EGFRvIII was more resistant to inhibition by gefitinib requiring a concentration of $2 \mu \mathrm{M}$ to reach a level comparable to that of the parental cell line (Figure 4A) The calculated IC $_{50}$ values for inhibition of EGFR- and EGFRvIII-mediated proliferation were 0.97 and $0.52 \mu \mathrm{M}$ for the lowand high-EGFR-expressing cell lines, respectively, and $7.58 \mu \mathrm{M}$ for the EGFRvIII-expressing cell line (Figure 4B).

Notably, low levels of gefitinib $(0.05-0.25 \mu \mathrm{M})$ resulted in a slight, but statistically significant $(P<0.05)$, increase in proliferation of EGFRvIII-expressing cells as compared to mock-treated cells (Figure 4A).

\section{Low concentrations of gefitinib increases the number of colonies of EGFRvIII-expressing cells}

To investigate the effect of gefitinib on EGFRvIII-mediated transformation colony formation assays were performed in semisolid agar. Results confirmed that in the absence of gefitinib NR6W and NR6M cells readily form colonies in soft agar, whereas NR6wtEGFR cells require $10 \mathrm{nM}$ EGF for weak colony formation (Figure 5). A gefitinib concentration above $0.1 \mu \mathrm{M}$ decreases the colony-forming ability of NR6W and NR6wtEGFR cells and a concentration of $1.5 \mu \mathrm{m}$ completely abolishes the ability of these cell lines to form colonies.

Surprisingly, gefitinib in the dose range from 0.1 to $0.5 \mu \mathrm{M}$ significantly facilitates, rather than abrogate, colony formation of NR6M cells. However, at a concentration of $2 \mu \mathrm{M}$ gefitinib completely blocks NR6M colony formation, as seen for the EGFR-expressing cell lines.

\section{Gefitinib-induced dimerisation of wt and mutant EGFR}

As can be seen in Figure 6C gefitinib induces the formation of EGFR and EGFRvIII homodimers at concentrations of $0.01 \mu \mathrm{M}$ and

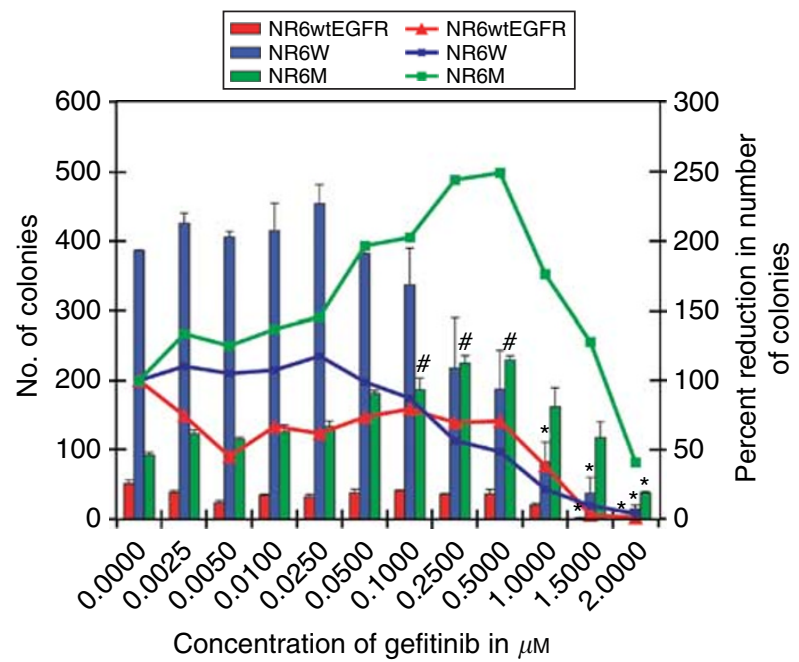

Figure 5 Growth of NR6 cells in soft agar in the presence and absence of gefitinib. (A) Graphical presentation of raw colony counts (left $y$-axis) and number of colonies plotted as a percentage of colonies in the untreated cells (right $y$-axis) for NR6, NR6wtEGFR, NR6W and NR6M cells treated with $0-2 \mu \mathrm{M}$ gefitinib and grown in soft agar for 18 days. NR6wtEGFR and NR6W were stimulated with 10 and $0.1 \mathrm{nM}$, respectively. This figure is representative of three independent experiments; bars, \pm s.e. ${ }^{\#} P<0.05$ (increase as compared to control treatment), $* P<0.05$ (decrease as compared to control treatment). 


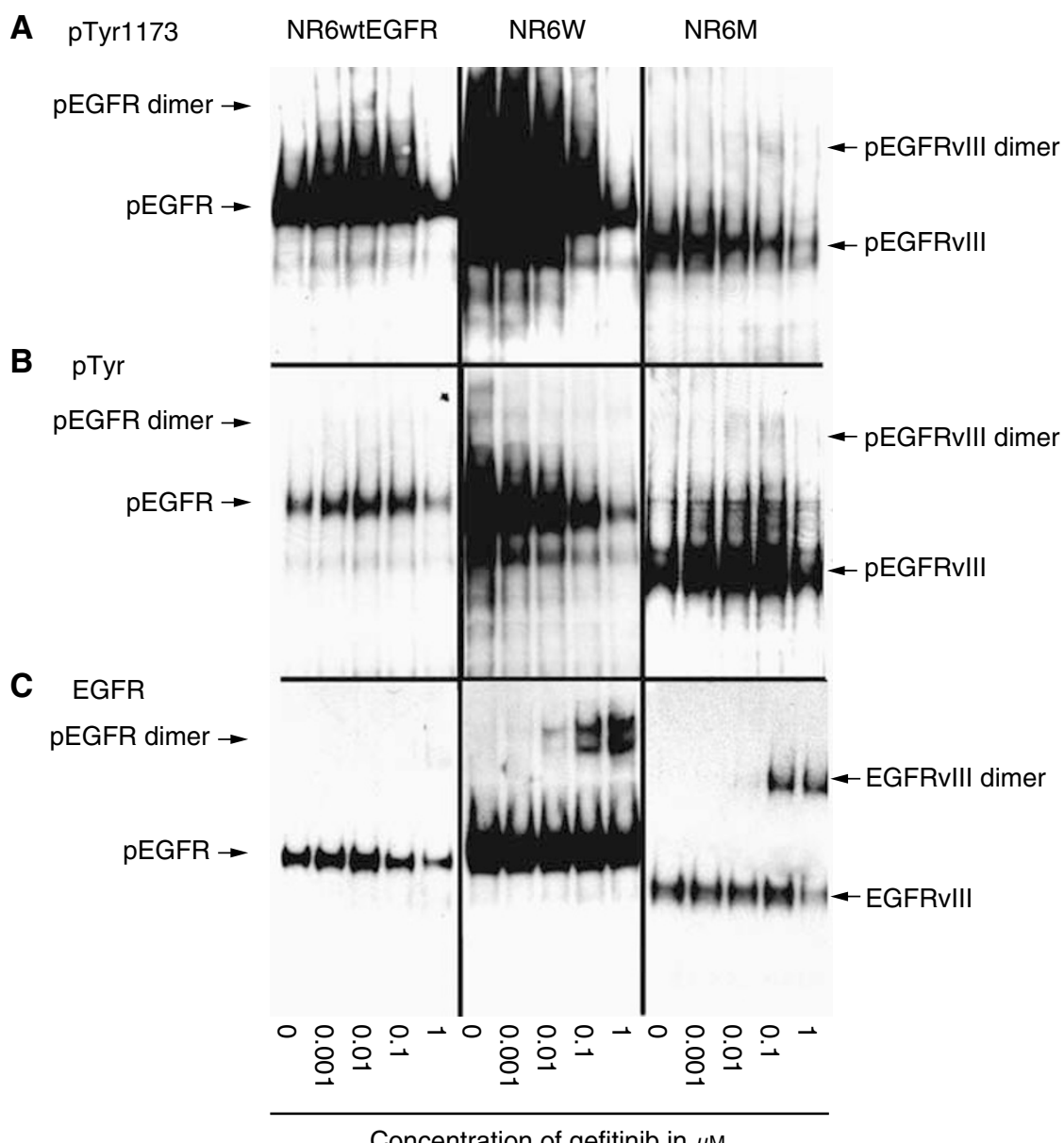

Figure 6 Dimerisation induction by gefitinib. Monolayer NR6wtEGFR, NR6W and NR6M cells were exposed to gefitinib for $24 \mathrm{~h}$ followed by exposure to the chemical crosslinker $\left(\mathrm{BS}^{3}\right)$ followed by immunoblot analysis using (A) anti-EGFR(pTyrl I73), (B) antiphospho-tyrosine (PY-20) and (C) anti-EGFR and antibodies.

above in the NR6W and NR6M cell lines. Furthermore, antiphosphotyrosine and antiphospho EGFR (Tyr1173) immunoblot analyses (Figure $6 \mathrm{~A}$ and $\mathrm{B}$ ) indicate that gefitinib in the concentration range $0.01-0.1 \mu \mathrm{M}$ increases the level of tyrosinephosphorylated EGFRvIII monomers and homodimers. A higher gefitinib concentration $(1 \mu \mathrm{M})$ decreases the phosphotyrosine load of both monomers and dimers to levels lower than the untreated controls. In contrast, although gefitinib induces EGFR homodimerisation in the high-EGFR-expressing cell line, a strong decrease in phosphotyrosine load is found for both the monomer and dimer bands (Figure 6). This indicates that the induction of activated dimers by gefitinib only takes place in cells expressing EGFRvIII. Of note, the band with a molecular weight approximating EGFRvIII detected in the NR6W and NR6wtEGFR cell lines is not EGFRvIII as confirmed using RT-PCR and antibodies specific for EGFRvIII (data not shown). Rather, it possibly represents a degraded form of EGFR, the level of which depends on the lysis buffer, the primary antibody and the homogenisation procedure.

\section{Durability of gefitinib inhibition in vitro}

A recent study has suggested that short-term treatment with gefitinib does not reduce phosphorylation of EGFRvIII and that repeated administration of gefitinib is necessary (Learn et al, 2004). Thus, to estimate the durability of gefitinib inhibition in vitro, an experiment was performed investigating the levels of EGFR/EGFRvIII and ERK phosphorylation in cells mock treated or treated with 0.1 or $2 \mathrm{~mm}$ of gefitinib and at the same time stimulated with $10 \mathrm{~nm}$ EGF for $10 \mathrm{~min}, 24,48$ and $72 \mathrm{~h}$ (Figure 7). The tyrosine phosphorylation site 1173 was selected, as it was the most durable of the phosphorylation sites. The results show that gefitinib rapidly and in a dose-dependent manner inhibits EGFR and ERK phosphorylations up to $72 \mathrm{~h}$ after EGF stimulation in both the high- and low-EGFR-expressing cell lines (Figure 7A and B). The decrease in EGFR levels, in the absence of gefitinib, in the low-EGFR-expressing cell line is due to receptor downregulation induced by EGF, a mechanism that appears not to be functioning in the NR6W and NR6M cell lines.

A concentration of $0.1 \mu \mathrm{m}$ gefitinib seems to increase EGFRvIII phosphorylation on residue 1173 and ERK phosphorylation after 48 and $72 \mathrm{~h}$ of treatment (Figure 7C). This could explain why gefitinib in low concentrations induce cell proliferation- and anchorage-independent growth. In contrast $2 \mu \mathrm{M}$ of gefitinib effectively inhibits both EGFRvIII and ERK phosphorylations in the NR6M cell line for up to $72 \mathrm{~h}$. It is noteworthy that the high concentration of gefitinib seems to induce degradation of both EGFR and EGFRvIII independent of EGF. This is particularly evident in the low-EGFR-expressing cell line where $0.1 \mu \mathrm{m}$ gefitinib inhibit EGF-mediated downregulation, presumably due to its inhibition of the Tyr1045 site, but fails to do so at a concentration of $2 \mu \mathrm{M}$ (Figure $7 \mathrm{~A}$ ). 
A

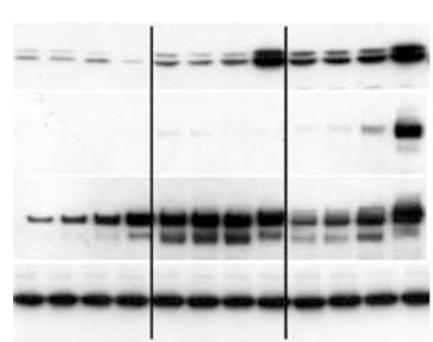

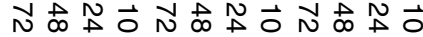

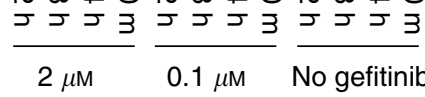

Gefitinib Gefitinib
B

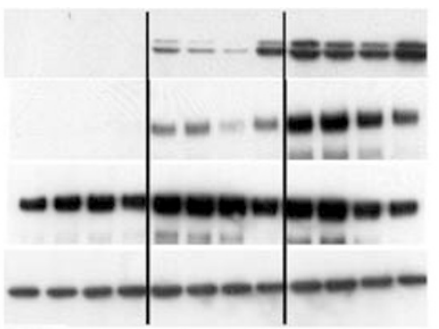

N ว $2 \frac{1}{2}$ ป

$2 \mu \mathrm{M}$

Gefitinib c

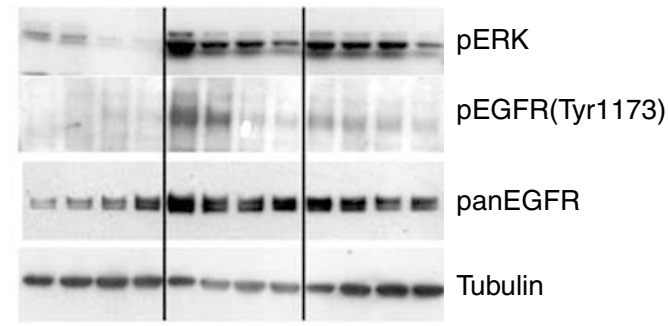

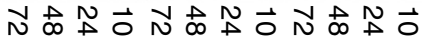

ン ン

$2 \mu \mathrm{M} \quad 0.1 \mu \mathrm{M} \quad$ No gefitinib

Gefitinib Gefitinib

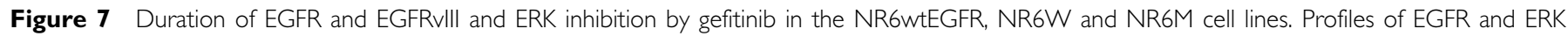
phosphorylations in the presence of varying concentrations of gefitinib and at varying time points in (A) the low-EGFR-expressing cell line NR6wtEGFR and (B) the high-EGFR-expressing cell line NR6W both stimulated with IO nM EGF. (C) Profiles of constitutive EGFRvlll phosphorylation and ERK in the presence of varying concentrations of gefitinib and at varying time points in the EGFRvlll-expressing cell line NR6M. The phosphorylations were determined by immunoblotting of whole cell lysates with antibodies that specifically recognise the phosphorylated species of ERK and EGFR-Tyr I 73. Total levels of EGFR, EGFRvIll and tubulin are also shown.

\section{DISCUSSION}

Phosphorylation of EGFR tyrosine residue 1173, AKT and ERK are least susceptible to inhibition by gefitinib

Gefitinib effectively inhibits phosphorylation of seven major tyrosine phosphorylation sites on EGFR and does so in cells expressing both low and high levels of EGFR. However, the effectiveness of inhibition of the individual sites varies with the Tyr1173 and Tyr992 being the more resistant in both cell lines. The levels of gefitinib sufficient to suppress EGFR phosphorylation are not sufficient to inhibit EGFRvIII phosphorylation. Average tyrosine phosphorylation of EGFRvIII being roughly four times more resistant to inhibition by gefitinib compared to the wt receptor with Tyr1148 and Tyr1173 being the more resistant sites. Thus, Tyr1173 appears to be the sturdiest tyrosine phosphorylation site in both EGFR and EGFRvIII requiring the highest concentration of gefitinib for inhibition. Tyr1173 is a major binding site for the adapter protein SHC and mediates activation of the RAS/RAF/MEK/ERK pathway (Batzer et al, 1994; Okabayashi et al, 1994). This is in concurrence with the observed persistence of ERK phosphorylation in the three cell lines even at high gefitinib concentrations.

As for ERK, the phosphorylation of AKT was resistant to gefitinib inhibition. The phosphorylation site(s) that mediates AKT phosphorylation is ill-defined, although Tyr1068 and Tyr1086 have been implicated through their binding to GRB2-associated binding protein 1 (GAB-1) (Rodrigues et al, 2000). As both Tyr1068 and Tyr1086 were sensitive to gefitinib inhibition it seems as if weakly phosphorylated receptors are able to facilitate AKT phosphorylation. The gefitinib-sensitive SRC ( $\mathrm{v}$-src sarcoma viral oncogene homolog) phosphorylation site Tyr845 on EGFR has been shown to mediate activation of STAT3, and indeed loss of EGF-dependent phosphorylation of STAT3 in response to gefitinib appears to be tightly coupled to suppression of EGFR autophosphorylation (Sato et al, 2003; Shao et al, 2003). Although EGFRvIII was phosphorylated on Tyr845, it was unable to induce STAT3 phosphorylation.

In general, the levels of gefitinib sufficient to inhibit the tyrosine phosphorylation sites on EGFR and EGFRvIII were insufficient to inhibit the downstream-signalling pathways, although phosphorylation of STAT3 was an exception. We believe that this is due to the amplification of the receptor signal seen by most signalling cascades (Mayawala et al, 2004). Targeting one or more kinases downstream of EGFR and EGFRvIII such as MEK, ERK or AKT could thus potentially increase the effectiveness of gefitinib. STAT3 is an exception as it binds directly to the receptor and following phosphorylation the protein translocates to the nucleus.

Epidermal growth factor receptorvIII-mediated cell proliferation and anchorage-independent growth is both resistant and stimulated by gefitinib

Like EGFRvIII phosphorylation and signalling, EGFRvIII-driven proliferation and anchorage-independent growth was found to be less sensitive to inhibition by gefitinib compared to EGFR. However, the level of gefitinib required for inhibition of these cellular properties was much higher than those needed for inhibition of receptor phosphorylation. The relative instability of inhibitor over extended periods at room temperature may explain the discrepancy between gefitinib levels that were needed for $50 \%$ inhibition of proliferation ( $\mathrm{IC}_{50}$ of $7.58 \mu \mathrm{M}$ ) and colony formation $\left(\mathrm{IC}_{50}\right.$ of $\left.2 \mu \mathrm{M}\right)$ and those that were needed for $50 \%$ inhibition of autophosphorylation (average $\mathrm{IC}_{50}$ of $0.091 \mu \mathrm{M}$ ).

Surprisingly, however, our results suggest not only that EGFRvIII-driven proliferation of NR6M cells is resistant to inhibition by gefitinib, but also appear to be enhanced by treatment with the inhibitor in the concentration range $0.05-$ $0.25 \mu \mathrm{M}$. A similar effect was observed for EGFRvIII-mediated anchorage-independent growth of NR6M cells, where colony formation was both resistant to and even stimulated by gefitinib in concentrations between 0.1 and $0.5 \mu \mathrm{M}$.

Although a novel finding with regard to gefitinib, similar inductions of proliferation and colony formation of both EGFRand EGFRvIII-expressing cells have been described in the literature with other quinazoline tyrosine kinase inhibitors (Montgomery, 2002; Li et al, 2003). Montgomery found that the EGFR-specific quinazoline inhibitor AG1478 at a concentration of $0.1 \mu \mathrm{M}$ stimulated colony formation of EGFRvIII-expressing cells (Montgomery, 2002). Similarly Li et al (2003) reported that PD153035 stimulated the proliferation of LN229/EGFR cells at $0.05 \mu \mathrm{M}$. Thus, this seems to be a general effect of EGFR-specific quinazoline inhibitors, when used at certain concentrations.

The mechanism by which quinazolines increase proliferation and anchorage-independent growth of EGFRvIII-expressing cells is uncertain. We did not see an effect on phosphotyrosine load of and 
signalling by EGFRvIII after $5 \mathrm{~h}$ of gefitinib inhibition (Figures 2 and 3). However, upon prolonged exposure ( $>24 \mathrm{~h}$ ) of EGFRvIIIexpressing cells to gefitinib in the concentration range $0.01-0.1 \mu \mathrm{M}$, EGFRvIII formed dimers and the phosphotyrosine load increased in both monomers and dimers. This is consistent with findings by Montgomery, who showed that AG1478 induced dimers of EGFRvIII in the concentration range $0.001-0.01 \mu \mathrm{M}$, and that dimers similarly had increased phosphotyrosine load (Montgomery, 2002). Addition of higher concentrations of both gefitinib and AG1478 maintain the level of EGFRvIII dimers, but the phosphotyrosine load decreases accordingly. We speculate that EGFR-specific quinazoline inhibitors in certain concentrations, depending on its stability and the level of receptor, capture EGFRvIII in its dimer form. As the gefitinib concentration gradually decreases over time, it reaches a level where the receptor captured in the complexes is able to transphosphorylate the dimer partner. This is further supported by the observation that gefitinib in a concentration of $0.1 \mu \mathrm{m}$ initially $(10 \mathrm{~min}-24 \mathrm{~h})$ inhibited EGFRvIII phosphorylations on Tyr1173 and ERK, but after $48 \mathrm{~h}$ increased their phosphorylations (Figure 7). Higher concentrations $(2 \mu \mathrm{M})$ effectively blocked EGFRvIII and ERK phosphorylations for up to $72 \mathrm{~h}$.

Although gefitinib was proficient at inducing dimers of EGFR in the high-EGFR-expressing cell line no increase in phosphotyrosine load could be detected. On the contrary gefitinib effectively inhibited phosphotyrosine load of both dimers and monomers.

Are the concentrations of gefitinib that stimulate EGFRvIII dimerisation, induce NR6M proliferation and anchorage-independent growth relevant in a clinical setting?

A recent study showed that daily oral dosing in the $400-$ $600 \mathrm{mg} \mathrm{day}^{-1}$ range result in mean plasma concentrations ranging from 478 to $620 \mathrm{ng} \mathrm{ml}^{-1}(1.07-1.39 \mu \mathrm{M})$ (Baselga et al, 2002). Assuming that the intratumour concentration equals the mean plasma concentration EGFRvIII phosphorylation and signalling should to be blocked, although cells are still proliferating at a higher rate than the control cells. However, if a lower daily dosing schema $\left(225 \mathrm{mg} \mathrm{day}^{-1}\right)$ is selected, the mean plasma concentration decreases to $160 \mathrm{ng} \mathrm{ml}^{-1}(0.36 \mu \mathrm{M})$ (Baselga et al, 2002). At this concentration EGFRvIII is able to induce phosphorylation of both ERK- and AKT- and EGFRvIII-mediated cell proliferation is largely unaffected. However, it is possible that by maintaining a daily high dosing scheme, the unwanted stimulation of EGFRvIII by gefitinib may be avoided, and this warrants further investigations in the clinical setting.

In summary this study shows that gefitinib blocks EGFR- and EGFRvIII-mediated phosphorylation and signalling, although higher concentrations are needed for effective inhibition of EGFRvIII. A similar resistance to gefitinib was observed on EGFRvIII-driven proliferation and anchorage-independent growth. In addition, our data provide evidence that long-term exposure of EGFRvIII-expressing cells to low concentrations of gefitinib augment EGFRvIII phosphorylation, signalling, cellular proliferation and anchorage-independent growth. Further studies are needed to investigate the implications of these findings in a more clinical setting.

\section{ACKNOWLEDGEMENTS}

AstraZeneca maker of Gefitinib and the Danish Cancer Society supported this work. The authors certify that they have not entered into any agreement that could interfere with their access to the data on the research, nor upon their ability to analyse the data independently, to prepare manuscripts, and to publish them. HSP has received research funding from AstraZeneca (maker of gefitinib).

\section{REFERENCES}

Alper O, Bergmann-Leitner ES, Bennett TA, Hacker NF, Stromberg K, Stetler-Stevenson WG (2001) Epidermal growth factor receptor signaling and the invasive phenotype of ovarian carcinoma cells. J Natl Cancer Inst 93: $1375-1384$

Arteaga CL, Johnson DH (2001) Tyrosine kinase inhibitors-ZD1839 (Iressa). Curr Opin Oncol 13: $491-498$

Baselga J, Rischin D, Ranson M, Calvert H, Raymond E, Kieback DG, Kaye SB, Gianni L, Harris A, Bjork T, Averbuch SD, Feyereislova A, Swaisland H, Rojo F, Albanell J (2002) Phase I safety, pharmacokinetic, and pharmacodynamic trial of ZD1839, a selective oral epidermal growth factor receptor tyrosine kinase inhibitor, in patients with five selected solid tumor types. J Clin Oncol 20: $4292-4302$

Batra SK, Castelino-Prabhu S, Wikstrand CJ, Zhu X, Humphrey PA, Friedman HS, Bigner DD (1995) Epidermal growth factor ligandindependent, unregulated, cell- transforming potential of a naturally occurring human mutant EGFRvIII gene. Cell Growth Differ 6: $1251-1259$

Batzer AG, Rotin D, Urena JM, Skolnik EY, Schlessinger J (1994) Hierarchy of binding sites for Grb2 and Shc on the epidermal growth factor receptor. Mol Cell Biol 14: 5192-5201

Damstrup L, Wandahl PM, Bastholm L, Elling F, Skovgaard PH (2002) Epidermal growth factor receptor mutation type iii transfected into a small cell lung cancer cell line is predominantly localized at the cell surface and enhances the malignant phenotype. Int J Cancer 97: 7-14

Garcia dPI, Adams GP, Sundareshan P, Wong AJ, Testa JR, Bigner DD, Weiner LM (1993) Expression of mutated epidermal growth factor receptor by non-small cell lung carcinomas. Cancer Res 53: 3217-3220

Ge H, Gong X, Tang CK (2002) Evidence of high incidence of EGFRvIII expression and coexpression with EGFR in human invasive breast cancer by laser capture microdissection and immunohistochemical analysis. Int J Cancer 98: $357-361$

Hansen LT, Lundin C, Helleday T, Poulsen HS, Sorensen CS, Petersen LN, Spang-Thomsen M (2003) DNA repair rate and etoposide (VP16) resistance of tumor cell subpopulations derived from a single human small cell lung cancer. Lung Cancer 40: 157-164

Huang HS, Nagane M, Klingbeil CK, Lin H, Nishikawa R, Ji XD, Huang CM, Gill GN, Wiley HS, Cavenee WK (1997) The enhanced tumorigenic activity of a mutant epidermal growth factor receptor common in human cancers is mediated by threshold levels of constitutive tyrosine phosphorylation and unattenuated signaling. $J$ Biol Chem 272: 2927- 2935

Learn CA, Hartzell TL, Wikstrand CJ, Archer GE, Rich JN, Friedman AH, Friedman HS, Bigner DD, Sampson JH (2004) Resistance to tyrosine kinase inhibition by mutant epidermal growth factor receptor variant III contributes to the neoplastic phenotype of glioblastoma multiforme. Clin Cancer Res 10: 3216-3224

Li B, Chang CM, Yuan M, McKenna WG, Shu HK (2003) Resistance to small molecule inhibitors of epidermal growth factor receptor in malignant gliomas. Cancer Res 63: 7443-7450

Lynch TJ, Bell DW, Sordella R, Gurubhagavatula S, Okimoto RA, Brannigan BW, Harris PL, Haserlat SM, Supko JG, Haluska FG, Louis DN, Christiani DC, Settleman J, Haber DA (2004) Activating mutations in the epidermal growth factor receptor underlying responsiveness of non-small-cell lung cancer to gefitinib. N Engl J Med 350: 2129-2139

Mayawala K, Gelmi CA, Edwards JS (2004) MAPK cascade possesses decoupled controllability of signal amplification and duration. Biophys $J$ 87: L01 - L02

Montgomery RB (2002) Antagonistic and agonistic effects of quinazoline tyrosine kinase inhibitors on mutant EGF receptor function. Int J Cancer 101: $111-117$

Moscatello DK, Holgado-Madruga M, Godwin AK, Ramirez G, Gunn G, Zoltick PW, Biegel JA, Hayes RL, Wong AJ (1995) Frequent expression of a mutant epidermal growth factor receptor in multiple human tumors. Cancer Res 55: 5536-5539

O’Rourke DM, Nute EJ, Davis JG, Wu C, Lee A, Murali R, Zhang HT, Qian X, Kao CC, Greene MI (1998) Inhibition of a naturally occurring EGFR 
oncoprotein by the p185neu ectodomain: implications for subdomain contributions to receptor assembly. Oncogene 16: 1197-1207

Okabayashi Y, Kido Y, Okutani T, Sugimoto Y, Sakaguchi K, Kasuga M (1994) Tyrosines 1148 and 1173 of activated human epidermal growth factor receptors are binding sites of Shc in intact cells. J Biol Chem 269: $18674-18678$

Olapade-Olaopa EO, Moscatello DK, MacKay EH, Horsburgh T, Sandhu DP, Terry TR, Wong AJ, Habib FK (2000) Evidence for the differential expression of a variant EGF receptor protein in human prostate cancer. Br J Cancer 82: 186 - 194

Paez JG, Janne PA, Lee JC, Tracy S, Greulich H, Gabriel S, Herman P, Kaye FJ, Lindeman N, Boggon TJ, Naoki K, Sasaki H, Fujii Y, Eck MJ, Sellers WR, Johnson BE, Meyerson M (2004) EGFR mutations in lung cancer: correlation with clinical response to gefitinib therapy. Science 304: 1497-1500

Pedersen MW, Meltorn M, Damstrup L, Poulsen HS (2001) The type III epidermal growth factor receptor mutation. Biological significance and potential target for anti-cancer therapy. Ann Oncol 12: 745-760

Pedersen MW, Tkach V, Pedersen N, Berezin V, Poulsen HS (2004) Expression of a naturally occurring constitutively active variant of the epidermal growth factor receptor in mouse fibroblasts increases motility. Int J Cancer 108: $643-653$
Peghini PL, Iwamoto M, Raffeld M, Chen YJ, Goebel SU, Serrano J, Jensen RT (2002) Overexpression of epidermal growth factor and hepatocyte growth factor receptors in a proportion of gastrinomas correlates with aggressive growth and lower curability. Clin Cancer Res 8: 2273-2285

Rodrigues GA, Falasca M, Zhang Z, Ong SH, Schlessinger J (2000) A novel positive feedback loop mediated by the docking protein Gabl and phosphatidylinositol 3-kinase in epidermal growth factor receptor signaling. Mol Cell Biol 20: $1448-1459$

Sato K, Nagao T, Iwasaki T, Nishihira Y, Fukami Y (2003) Src-dependent phosphorylation of the EGF receptor Tyr-845 mediates Stat-p21waf1 pathway in A431 cells. Genes Cells 8: $995-1003$

Shao H, Cheng HY, Cook RG, Tweardy DJ (2003) Identification and characterization of signal transducer and activator of transcription 3 recruitment sites within the epidermal growth factor receptor. Cancer Res 63: 3923-3930

Tang CK, Gong XQ, Moscatello DK, Wong AJ, Lippman ME (2000) Epidermal growth factor receptor vIII enhances tumorigenicity in human breast cancer. Cancer Res 60: 3081-3087

Wells A, Welsh JB, Lazar CS, Wiley HS, Gill GN, Rosenfeld MG (1990) Ligand-induced transformation by a noninternalizing epidermal growth factor receptor. Science 247: $962-964$ 